\title{
Maximum Peak-Gain Margin 2DOF-IMC Tuning for a 2DOF-PID Filter Set Point Controller under Parametric Uncertainty Model
}

\author{
Nur Hidayah ${ }^{1}$, Juwari ${ }^{2}$, and Renanto Handogo ${ }^{2}$
}

\begin{abstract}
The specification of controller setting for a standard controller typically requires a trade-off between set point tracking and disturbance rejection. For this reason two simple strategies can be used to adjust the set point and disturbance responses independently. These strategies are referred to as controllers with two degree of freedom. Unfortunately, the tuning parameters in the case of model uncertainty at two degree of freedom structure controller is difficult to obtain. Juwari et al (2013) has introduced maximum peak-gain margin (Mp-GM) tuning method to obtain setting parameter of two degree of freedom structure controller based on model uncertainty. This tuning method are able to obtain the good controller parameter even under processes uncertainties on standard two degree of freedom (was abbreviated as 2DOF) IMC. This research will be conducted on development maximum peak-gain margin tuning method for a two degree of freedom PID filter set point structure controller. The simulation results show that the maximum peak gain margin tuning method can give a good target set point tracking, disturbance rejection and robustness in system a 2DOF-PID filter set point controller.
\end{abstract}

Keywords — $\longrightarrow$ wo degree of freedom structure controller, IMC, PID, maximum peak, gain margin.

\section{INTRODUCTION}

A chemical industry generally consists of many unit operations, which must be operated on specific conditions such as temperature, pressure and flow. This operating condition(s) is maintained for the purpose of safety and product quality. To acquire this condition necessary circuit system control being able to control production remained according to the condition desired. Various designs controller were developed to obtain this goal. Proportional Integral Derivative (PID) controller has been used in the industry since 1940's for this purpose because the PID controller uses a simple algorithm. PID control system are widely used as basic control technology for industrial control systems today, due to its well known as simple PID control structure [1]. Although the development of PID controller rapidly but still has not produce maximum results especially to a process with time a delay. This is due to disturbance are not detected immediately (detected until certain time with delay), control actions based on the delay is not accordance with the purpose of information so need some time to determine its effects on the process. To overcome this weakness, Morari and associates (Garcia, Zafiriou, Rivera and Skogestad) develop new structure controller that called as internal model control (IMC) controller. The design of this structure take advantage of the approach of the process model to control the system [2].

A controller $G_{c}(s)$ is used to control the process, $G_{p}(s)$. Suppose $G_{p m}(s)$ is a model of $G_{p}(s)$. By setting $G_{c}$ (s) to be the inverse of the model of the process, $G_{c}(s)=$ $\mathrm{G}_{\mathrm{pm}}(\mathrm{s})^{-1}$, and if $\mathrm{G}_{\mathrm{p}}(\mathrm{s})=\mathrm{G}_{\mathrm{pm}}(\mathrm{s})$. Then it is clear that the output will always be equal to the set point. So the ideal control performance can be obtained without feedback when we have complete knowlodge about the process

\footnotetext{
${ }^{1}$ Nur Hidayah is with Undergraduated Student of Chemical Engineering Department, Faculty of Industry Engineering, Institut Teknologi Sepuluh November, Surabaya, 60111, Indonesia. E-mail: Day.chemeng@gmail.com.

${ }^{2}$ Juwari and Renanto Handogo are with Department of Chemical Engineering, Faculty of Industry Engineering, Institut Teknologi Sepuluh November, Surabaya, 60111, Indonesia.
}

being controlled and there is no disturbance enters to process. In practice, unknown disturbance can affected the system so IMC design cannot give fast response to case disturbance rejection [3].

Although 1DOF-IMC controller give a slow response to case disturbance rejection, this controller has advantage to tune PID controller. One of advantage of 1DOF-IMC is that can be analogous with PID controller (see Figure 2). The IMC structure is simplified to a conventional feedback structure and the algorithm then analogous to PID algorithm. There are many PID tuning method based on the principle of IMC $[2,4,12]$. This $G_{c}$ form then can be converted into PI/PID controller [4].

$$
G_{c}(s)=\frac{G_{c 1}(s)}{1-G_{p m} G_{c 1}(s)}
$$

The specification of controller settings for a standard controller typically requires a tradeoff between set point tracking and disturbance rejection. For most single loop controllers, disturbance rejection is more important than set point tracking, although one exception occurs when the set point is calculated by another controller. Thus, it is reasonable to tune the controller for satisfactory disturbance rejection, especially if it can be achieved without sacrificing set point tracking. Fortunately, two simple strategies can be used to adjust the set point and disturbance responses independently. These strategies are referred to as controllers with two degree of freedom [4]. The design of control systems is a multi-objective problem, so a two degree of freedom (abbreviated as 2DOF) control system naturally has advantages over a one degree of freedom (abbreviated as 1DOF) control system. This fact was already stated by Horowitz, but did not attract a general attention from engineers for a long time, it was only in 1984, two decades after Horowitz's work, that a research to exploit the advantages of the $2 \mathrm{DOF}$ structure for PID control has two sets of PID parameters, one can be used to optimize the performance of the command tracking, the other can be used to optimize the performance of the disturbance rejection [5].

The development of strategy control 2DOF-PID in 1984 was begin with research by Araki. There are 
several designs 2DOF-PID controller was generated for use in industry such as 2DOF-PID filter set point type, 2DOF-PID feedforward and 2DOF-PID feedback. A form of the two degree of freedom PID control system is show in Fig. 2 was developed by Araki et al. The 2DOFPID structure controller consist of two compensators $\mathrm{C}_{\mathrm{r}}(\mathrm{s})$ as controller for set point tracking and $\mathrm{C}_{\mathrm{y}}(\mathrm{s})$ as controller for disturbance rejection with $\mathrm{P}(\mathrm{s})$ as a transfer function of process. The close loop transfer function for the 2DOF-PID filter set point in equation (1)

$$
y=\frac{C_{r}(s) P(s) r+d}{1+C_{y}(s) P(s)}
$$

With controller algorithm for $\mathrm{C}_{\mathrm{r}}(\mathrm{s})$ and $\mathrm{C}_{\mathrm{y}}(\mathrm{s})$ were given in equation (2) and (3)

$$
\begin{aligned}
& C_{r}(s)=\frac{1+(1-a) \tau_{I}(s)+(1-\beta) \tau_{I}(s) \tau_{D}(s) d(s)}{1+\tau_{I}(s)+\tau_{I}(s) \tau_{D}(s) d(s)} \\
& C_{y}(s)=k_{p}\left[1+\frac{1}{\tau_{I} s}+\tau_{D}(s)\right]
\end{aligned}
$$

Where $\mathrm{k}_{\mathrm{c}}$ as a proportional gain controller, $\tau_{\mathrm{I}}$ as integral time constant, $\tau_{\mathrm{D}}$ as derivative time constant and $\alpha$ and $\beta$ variable as $2 \mathrm{DOF}$ structure control parameter. Variable constant value of $\alpha$ and $\beta$ given among $0 \leq(\alpha$ and $\beta) \leq 1$.

Based on equation (2), we can see that Araki add a filter function on the conventional PID controller which was used to control set point tracking in controller $\mathrm{F}(\mathrm{s})$. $\mathrm{C}^{\prime}$ (s) controller to control disturbance rejection use PID parallel controller [5]. In addition, Vilanova et al also developed another structure controller 2DOF-PID. This structure was implemented for control FOPDT and SOPDT process using PI/PID controller in set point tracking and disturbance rejection compensators [6].

For controller based on internal model, the developing two degree of freedom structure controller beginning since 1989 by Morari and Zafiriou. This structure is developed to overcome delays response of disturbance rejection in one degree of freedom IMC. The effect of a 2DOF-IMC structure is to include one lead lag transfer function to the feedback loop [2]. The Fig. 4 shows the 2DOF-IMC standard structure control consist of two compensators $G_{c 1}$ as the controller for set point and $G_{c 2}$ a controller for disturbance rejection. The close loop transfer function for the 2DOF-IMC standard in equation (5)

$$
y=\frac{\left(G_{p} G_{c 1}\right) y_{s p}+\left(1-G_{c 2} G_{p m}\right)}{1+G_{c 2}\left(G_{p}-G_{p m}\right)}
$$

With controller algorithm for $\mathrm{G}_{\mathrm{c} 1}$ and $\mathrm{G}_{\mathrm{c} 2}$ were given in equation (6) and (7)

$$
\begin{aligned}
G_{c 1} & =\frac{1}{k} \frac{\tau s+1}{\lambda_{1} s+1} \\
G_{c 2} & =\frac{1}{k} \frac{\tau s+1}{\left(\lambda_{1} s+1\right)\left(\lambda_{2} s+1\right)}
\end{aligned}
$$

Where $\mathrm{k}_{\mathrm{c}}$ as a proportional gain controller, $\lambda_{1}$ as parameter low pass filter time constant for set point tracking controller and $\lambda_{2}$ as parameter low pass filter for disturbance rejection controller.

Another variation of 2DOF-IMC was developed by Ibrahim Kaya in 2004. This structure was called as 2DOF-IMC Kaya. By using principle stability of gain and phase margin, this structure was developed to control the integrating process with small time delay. 2DOF-IMC Kaya was proposed to tune/design PD controller [7]. Besides IMC, another controller based on model like SP controller also begin to be developed in the form of $2 \mathrm{DOF}$ structure controller. Tain and Gao (1999) was designed 2DOF SP structure control for integrating process with large time delay. This structure was able to provide quick and stable response to disturbance rejection.

Many design 2DOF structure controller that was proposed was not followed with the research on the tuning method for this structure. Whereas, tuning method is one of the important part in controlling. The purpose of controller tuning is to determine the parameters of controller in order to ensure the time response of closeloop control system at the desired performance. Performance of controller is considered good if the controlled variable is always at the desired set point [8]. The parameters tuning is very difficult to be performed under the case of model uncertainty, where there are inaccuracies between model and real plant. Model variation of real parameter affecting to plant operation, the inherent non-linearity of the process, the experiment identification of the process, and the mathematical model development. The most tuning method was proposed for 2DOF structure controller is still limited for controlling process with perfect models. The perfect models here means that the transfer function process and a model considered the same. Method tuning for PI, PD or PID controller was proposed by Araki and Taghuchi for the different 2DOF-PIDstructure controller like 2DOF-PID filter set point type, 2DOF-PID feedforward and 2DOFPID feedback. Unfortunately, the tuning method was proposed by Araki and Taghuci did not provide an analytical explanation for parameter controller and there are not definite guarantee that system control can give the stability and robustly response [9].

Another tuning method for 2DOF-PID structure was developed by Miluse and Antonin. They are proposed a new tuning with using multiplication dominant pole principle on the sensitivity function and complementary sensitivity function. Nevertheless, this tuning method still being developed for integrating process with small dead time. Besides that, this tuning method involving certain weighting factor on the proportional and derivative part on the set point tracking and disturbance rejection controller [10]. Vilanova et al was proposed another method tuning for 2DOF-PID structure controller. This method was proposed using analytical approximation. This method was called as analytical robust tuning (ART). ART method able to give a stability and robustly response in control case with process FOPDT and SOPDT using PI/PID controller. Unfortunately, this method also use weighting factor for determine parameter control in set point tracking controller [6].

Method tuning for 2DOF IMC were mostly still developed for controling the process with perfect model. One of the researchers developed a tuning method to the case of uncertainties is Brosilow and Joseph (2001). They are using the principle of resonant peak from complementary sensitivity function to develop tuning method on 2DOF-IMC structure controller. 
Unfortunately this method only used for 2DOF-IMC structure controller [11]. Hence by using Maximum peak principle was proposed by Brosilow and Stryczek et al which develop IMCTUNE that can was implemented not only on 1DOF-IMC and 2DOF-IMC but also at other structure controller such as 1DOF-PID, 2DOF-PID as well as MSF (Model State Feedback) controller. The weakness of this method was use of the partial sensitivity function that derived from the transfer function disturbance that is difficult to be modeled [12]

The newest, Juwari et all has introduced maximum peak-gain margin (Mp-GM) tuning method to obtain setting parameter of two degree of freedom structure controller based on model uncertainty. Maximum peak (Mp) is used for tuning of set point tracking controller $\left(\mathrm{Gc}_{1}\right)$ and GM (gain margin) is used for tuning of disturbance rejection $\left(\mathrm{Gc}_{2}\right)$. Then the proposed tuning method is denoted as Mp-GM method. The tuning is determined based on worst case of an uncertainty process. Three parameters are set to obtain the optimal controller response, these parameters are $\lambda_{1}, \lambda_{2}$ and $\alpha$.

The Mp-GM tuning method consist of three step. The first step is determining the worst case of uncertainty model. Worst case is conditions permitting the response control be unstable. The worst case can be found from the limit of the uncertainty model in terms of upper and lower on process model parameters. This condition usually occurs at the uncertainty model with the larger (upper limit) steady state gain process, the larger (upper limit) time delay and the smaller (lower limit) process time constant. The worst case can be identified at the biggest maximum value of magnitude of frequency response of complementary sensitivity function. When determining the worst case, time filter constant $(\lambda)$ value is set equal to the time delay of no error in the model.

The second step is specifying the parameter of set point controller $\left(\mathrm{G}_{\mathrm{c} 1}\right)$ using complementary sensitivity function of 1DOF-IMC structure, based on the maximum peak stability criterion. By using the algorithm $G_{c 1}=\frac{1}{k} \frac{\tau s+1}{\lambda_{1} s+1}$, where $\mathrm{k}$ is gain process, $\tau$ is time constant process and $\lambda_{1}$ is filter time constant parameter will be determined parameter $\lambda_{1}$ as parameter set point controller. The filter time constant parameter obtained by looping the value of $\lambda_{1}$ (filter time constant $\mathrm{Gc}_{1}$ ) in calculating complementary sensitivity function so acquired max $|T(j \omega)|=1.05$ in the range of frequency $\omega=10^{-3}$ to $10^{3}$. For the first looping, $\lambda_{1}$ is set equal to the time delay $(\theta)$ of no error in the model divided by 20 .

The third step is obtaining parameter of disturbance rejection controller $\left(\mathrm{G}_{\mathrm{c} 2}\right)$ using transfer function open loop of 2DOF structure controller based on the gain margin criteria. The disturbance rejection parameter obtained by looping the value of $\alpha$ in calculating transfer function open loop so acquired $\mathrm{GM}=2.4$. For the first looping, $\alpha$ is set equal to filter time constant parameter disturbance rejection controller $\left(\lambda_{2}\right)$ by setting ratio of $\lambda_{2}$ to $\lambda_{1}$ as much as 0.9 . This calculation using algorithm $G_{c 2}=G_{c 1} \frac{1}{k} \frac{\alpha s+1}{\lambda_{2} s+1}$, where $\mathrm{k}$ is gain process, $\tau$ is time constant process, $\lambda_{2}$ and $\alpha$ are filter time constant parameter and lead parameter at disturbance rejection controller respectively [13].
This tuning method are able to obtain the good controller parameter even under process uncertainties on standard two degree of freedom IMC structure control. The stability and robust Mp-GM tuning method has potential to be implemented into other 2DOF structure controller, especially 2DOF PID controller. This paper study analytical procedure of implementation of Mp-GM 2DOF-IMC tuning method to 2DO-PID structure controller under process uncertainties. This research will be conducted on development maximum peak-gain margin 2DOF-IMC tuning method for a two degree of freedom PID filter set point structure controller.

As a 1DOF-IMC structure, 2DOF-IMC structure also have an advantage that it can be analogous with 2DOFPID structure controller (see Figure. 5). Tuning of 2DOF-PID based on 2DOF IMC for integrator and dead time process is proposed by Zhang et al [14]. Figure.5 shows the controller $G_{\mathrm{c} 1}$ as controller set point tracking and $\mathrm{G}_{\mathrm{c} 2}$ as controller disturbance rejection of 2DOF-IMC structure control can be transformed equivalently to controller $\mathrm{F}(\mathrm{s})$ as controller set point tracking and $\mathrm{C}^{\prime}(\mathrm{s})$ as controller disturbance rejection of 2DOF-PID filter set point. From Figure. 5 we can obtain [14]

$$
\begin{aligned}
& y=\frac{\left(G_{p} G_{c 1}\right) r+\left(1-G_{c 2} G_{p m}\right) d}{1+G_{c 2}\left(G_{p}-G_{p m}\right)} \\
& F(s)=\frac{G_{c 1}(s)}{G_{c 2}(s)} \\
& C^{\prime}(s)=\frac{G_{c 2}(s)}{1-G_{p m} G_{c 2}(s)}
\end{aligned}
$$

\section{METHOD}

Procedure to implementation Mp-GM 2DOF-IMC tuning method for a 2DOF-PID structure controller done follow up step tuning developed by Zhang et al (2006) and Juwari et all (2013). Based on equation (9) and (10) was derived by Zhang et all, we can obtain transfer function of controller $\mathrm{F}(\mathrm{s})$ and $\mathrm{C}^{\prime}(\mathrm{s})$ of 2DOF-PID structure controller. The transfer functions models of case FOPDT process can be given as

$G_{p m}=\frac{K}{\tau s+1} e^{-\theta}$

To obtain form of PID regulator from IMC controller, the dead time of process model $G_{p m}$ will be approximated by Taylor series approximation that given in equation (12)

$e^{-\theta}=1-\theta(s)$

Based on Mp-GM tuning was proposed by Juwari et al, transfer functions for $\mathrm{G}_{\mathrm{c} 1}$ as controller set point tracking and $\mathrm{G}_{\mathrm{c} 2}$ as controller disturbance rejection can be obtained by equations (13) and (14) respectively.

$$
\begin{aligned}
G_{c 1} & =\frac{1}{k} \frac{\tau_{p} s+1}{\lambda_{1} s+1} \\
G_{c 2} & =G_{c 1} \frac{\alpha s+1}{\lambda_{2} s+1}
\end{aligned}
$$


From equations $(9) \sim(14)$, we can obtain transfer function controller $\mathrm{F}(\mathrm{s})$ as set point tracking controller and c'(s) as controller of disturbance rejection of 2DOFPID filter set point by equations (15) and (16) respectively

$$
\begin{aligned}
& F(s)=\frac{\lambda_{2} s+1}{\alpha s+1} \\
& C^{\prime}(s)=\frac{1}{k} \frac{\alpha \tau s^{2}+(\alpha+\tau) s+1}{\left(\lambda_{1} \lambda_{2}+\alpha \theta\right) s^{2}+\left(\lambda_{1}+\lambda_{2}-\alpha+\theta\right) s}
\end{aligned}
$$

$\mathrm{C}^{\prime}(\mathrm{s})$ controller that having a more complex transfer function than $\mathrm{F}(\mathrm{s})$ controller, it will be approached with a variety of PID controller such as PID parallel plus low pass filter, PID parallel plus derivative filter and PID series plus derivative filter. The form of the $C^{\prime}(s)$ controller approached with PID parallel plus derivative filter can be given by equation (17)

$$
C^{\prime}(s)=K_{c}\left(1+\frac{1}{\tau_{I} s}+\tau_{d} s\right) \frac{1}{\tau_{f} s+1}
$$

Where;

$$
\begin{gathered}
K_{c}=\frac{\alpha+\tau}{k\left(\lambda_{1}+\lambda_{2}+\theta-\alpha\right)} \\
\tau_{I}=\alpha+\tau \\
\tau_{d}=\frac{2 \tau \alpha}{(\alpha+\tau)} \\
\tau_{f}=\frac{\lambda_{1} \lambda_{2}+\alpha \theta}{\left(\lambda_{1}+\lambda_{2}+\theta-\alpha\right)}
\end{gathered}
$$

The form of the C'(s) controller approached with PID parallel plus derivative filter can be given by equation (18)

$$
C^{\prime}(s)=k_{C}\left(1+\frac{1}{\tau_{I} S}+\frac{\tau_{D} S}{A \tau_{D} s+1}\right)
$$

This equations will produce equation for $\mathrm{k}_{\mathrm{c}}, \tau_{\mathrm{I}}, \tau_{\mathrm{D}}$ and $\mathrm{A}$ parameter was given as

$$
\begin{aligned}
& k_{c}=\frac{\left(\lambda_{1}+\lambda_{2}+\theta-\alpha\right)(\alpha+\tau)-\left(\lambda_{1} \lambda_{2}+\alpha \theta\right)}{k\left(\lambda_{1}+\lambda_{2}+\theta-\alpha\right)^{2}} \\
& \tau_{I}=\alpha+\tau-\left(\frac{\lambda_{1} \lambda_{2}+\alpha \theta}{\left(\lambda_{1}+\lambda_{2}+\theta-\alpha\right)}\right) \\
& \tau_{D}=\left(\frac{\left(\lambda_{1}+\lambda_{2}+\theta-\alpha\right)}{\left(\lambda_{1}+\lambda_{2}+\theta-\alpha\right)(\alpha+\tau)-\left(\lambda_{1} \lambda_{2}+\alpha \theta\right)}\right)-\left(\frac{\lambda_{1} \lambda_{2}+\alpha \theta}{\left(\lambda_{1}+\lambda_{2}+\theta-\alpha\right)}\right) \\
& A=\frac{\lambda_{1} \lambda_{2}+\alpha \theta}{\tau_{D}\left(\lambda_{1}+\lambda_{2}+\theta-\alpha\right)}
\end{aligned}
$$

The form of the C'(s) controller approached with PID series plus derivative filter can be given by equation (19)

$C^{\prime}(s)=k_{C}\left(1+\frac{1}{\tau_{I} S}+\frac{\tau_{D^{S}}}{A \tau_{D^{S}}+1}\right)$

For determine parameter controller on the PID series with derivative filter will be approached with two different equation which is at $\tau_{\mathrm{I}}=\tau$ and $\tau_{\mathrm{D}}=\tau$. The approximation with $\tau_{\mathrm{I}}=\tau$ at equation (19) will produce $\mathrm{k}_{\mathrm{c}}, \tau_{\mathrm{I}}, \tau_{\mathrm{D}}$ and A parameter was given as

$$
\begin{aligned}
& k_{c}=\frac{\tau}{k\left(\lambda_{1}+\lambda_{2}+\theta-\alpha\right)} \\
& \tau_{I}=\tau \\
& \tau_{D}=\alpha \quad \\
& \qquad=\frac{\lambda_{1} \lambda_{2}+\alpha \theta}{\alpha\left(\lambda_{1}+\lambda_{2}+\theta-\alpha\right)}
\end{aligned}
$$

And when the approximation with $\tau_{\mathrm{D}}=\tau$, the equations (19) will produce $\mathrm{k}_{\mathrm{c}}, \tau_{\mathrm{I}}, \tau_{\mathrm{D}}$ and A parameter was given as

$$
\begin{aligned}
k_{c} & =\frac{\alpha}{k\left(\lambda_{1}+\lambda_{2}+\theta-\alpha\right)} \\
\tau_{I} & =\alpha \\
\tau_{D} & =\tau \\
& A=\frac{\lambda_{1} \lambda_{2}+\alpha \theta}{\tau\left(\lambda_{1}+\lambda_{2}+\theta-\alpha\right)}
\end{aligned}
$$

The parameter $\lambda_{1}, \lambda_{2}$ and $\alpha$ will produce from the MpGM tuning method was proposed by Juwari et all using complementary sensitivity function 1DOF-IMC (gives by equation (19)) structure for determine $\lambda_{1}$ (parameter filter time constant for set point tracking controller). Parameter $\lambda_{2}$ (parameter filter time constant for disturbance rejection controller) and $\alpha$ (lead parameter for disturbance rejection controller) will produce using GM principle from transfer function open loop 2DOFIMC (given by equations (20)) structure control. The complete step of Mp-GM tuning method have described in the previous section.

$$
\begin{aligned}
& T(j \omega)=\frac{G_{c} G_{p}}{1+G_{c}\left(G_{p}-G_{p m}\right)} y_{s p} \\
& G_{c 2}\left(G_{p}-G_{p m}\right)
\end{aligned}
$$

\section{RESULT AND DISCUSSION}

Three examples FOPDT case are considered to illustrate the implementation of the Mp-Gm 2DOF-IMC tuning method for 2dof-pid filter set point structure control. The examples cover FOPDT cases model with $\frac{\theta}{\tau}<1, \frac{\theta}{\tau}>1$ with $\theta<1$ and $\tau<1$ and $\frac{\theta}{\tau}>1$, where was used $(\theta>1$ and $\tau>1)$. For the FOPDT case model with $\frac{\theta}{\tau}<1$ is adopted from Vilanova et al. [15]. The FOPDT model with $\frac{\theta}{\tau}<1$ is described as below.

$G_{p}=\frac{K}{\tau s+1} e^{-\theta s}$

With upper and lower limit uncertainties parameter for controller as;

$0.8 \leq k \leq 1.2$,

$2.4 \leq \tau \leq 3.6$ and

$1.2 \leq \theta \leq 1.8$ 
And transfer function for process model an disturbance given on equation (23) and (24).

$G_{p m}=\frac{1}{3 s+1} e^{-1.5 s}$

$G_{d}=\frac{0.5}{2 s+1}$

For FOPDT case with $\frac{\theta}{\tau}<1$ obtained transfer function worst case process to be controlled having value $\mathrm{k}=1.2$, $\tau=2.4$ and $\theta=1.8$. The parameter transfer function process model was given as $\mathrm{k}=1, \tau=3$ and $\theta=1.5$. by using 2DOFIMC structure controller was tuned by MpGM tuning method then obtained parameter value for $\mathrm{G}_{\mathrm{c} 1}$ as controller set point tracking and $\mathrm{G}_{\mathrm{c} 2}$ as controller disturbance rejection is $\lambda_{1}=1.851, \lambda_{2}=1.6659$ and $\alpha=$ 3.0459. The parameter value of process model and MpGM 2DOF-IMC tuning was substituted to equation (17)(19) to obtain parameter value for variety PID controller. The complete result of calculation PID controller parameter in the FOPDT case with $\frac{\theta}{\tau}<1$ can be seen in Table. 1 .

The parameter have been obtained for each PID controller design then used to control the worst case process. The simulation was done using Simulink software. Input control set point in form of signals step that began in time 0 with magnitude of 1 . The disturbance enters the system at time 75 with magnitude of 0.5 .

For the FOPDT case model with $\frac{\theta}{\tau}>1$ with $\theta<$ 1 and $\tau<1$ is adopted from Chang et al. [16]. The FOPDT model with $\frac{\theta}{\tau}>1$ with $\theta<1$ and $\tau<1$ is described as below

$G_{p}=\frac{K}{0.2 s+1} e^{-\theta s}$

With upper and lower limit uncertainties parameter for controller as

$14.96 \leq k \leq 22.44$,

$0.4 \leq \theta \leq 0.6$

And transfer function for process model was given by equation (26)

$G_{p}=\frac{18.7}{0.2 s+1} e^{-0.5 s}$

For FOPDT case with $\frac{\theta}{\tau}>1(\theta<1$ and $\tau<1)$ obtained transfer function worst case process to be controlled having value $\mathrm{k}=22.44, \tau=0.2$ dan $\theta=$ 0.6 .The parametric transfer function process model was given as $\mathrm{k}=18.7, \tau=0.2$ and $\theta=0.5$.

Using 2DOF IMC structure controller was tuned by Mp-GM tuning method then obtained parameter value for $\mathrm{G}_{\mathrm{c} 1}$ as controller set point tracking and $\mathrm{G}_{\mathrm{c} 2}$ as controller disturbance rejection is $\lambda_{1}=0.524, \lambda_{2}=$ 0.4716 and $\alpha=1.0216$. The parameter value of process model and Mp-GM 2DOF-IMC tuning was substituted to equation (17)-(19) to obtain parameter value for variety PID controller. The complete result of calculation PID controller parameter in the FOPDT case with $\frac{\theta}{\tau}<1$ can be seen in Table. 2 .
The parameter have been obtained for each PID controller design then used to control the worst case process. The simulation was done using Simulink software. Input control set point in form of signals step that began in time 0 with magnitude of 1 . The disturbance enters the system at time 25 with magnitude of 0.5 .

Consider a pulp and paper processes industry, Nancy et.al developed the following dynamics equations [17] with $\frac{\theta}{\tau}>1$ (where was used $\theta>1$ and $\left.\tau>1\right)$ :

$G_{p}=\frac{K}{\tau s+1} e^{-\theta s}$

With upper and lower limit uncertainties parameter for controller as

$-0.0488 \leq k \leq-0.0326$,

$3.072 \leq \tau \leq 4.608$ and

$5.472 \leq \theta \leq 8.208$

And transfer function for process model was given by equation (28)

$G_{p}=\frac{-0.0407}{3.84 s+1} e^{-6.84 s}$

For FOPDT case with $\frac{\theta}{\tau}>1(\theta>1$ and $\tau>1)$ obtained transfer function worst case process to be controlled having value $\mathrm{k}=-0.0488, \tau=4.6080$ and $\theta=$ 8.2080. The parametric transfer function process model was given as $\mathrm{k}=-0.0407, \tau=3.84$ and $\theta=6.84$. By using 2DOF IMC structure controller was tuned by MpGM tuning method then obtained parameter value for $\mathrm{G}_{\mathrm{c} 1}$ as controller set point tracking and $\mathrm{G}_{\mathrm{c} 2}$ as controller disturbance rejection is $\lambda_{1}=8.433, \lambda_{2}=7.5897$ and $\alpha=$ 18.4597. The parameter value of process model and $\mathrm{Mp}$ GM 2DOF-IMC tuning was substituted to equation (17)(19) to obtain parameter value for variety PID controller. The complete result of calculation PID controller parameter in the FOPDT case with $\frac{\theta}{\tau}<1$ can be seen in Table 3.

The parameter have been obtained for each PID controller design then used to control the worst case process. The simulation was done using Simulink software. Input control set point in form of signals step that began in time 0 with magnitude of 1 . The disturbance enters the system at time 375 with magnitude of 0.5 .

From Figure 6 8 we can see that all form of PID controllers that are used give the response toward stability though with different time periods in all FOPDT case with value variety of $\theta / \tau$. FOPDT case with ratio $\frac{\theta}{\tau}<1$ produce fastest response with small settling time than another case in this paper. This is because at the FOPDT case with ratio $\frac{\theta}{\tau}<1$ has a big gain process value with small time constant process than another FOPDT case that is used. Based on IAE value and time period for achieve a stability response, the used of PID series plus derivative filter give the better result than another variety of PID controller, both with approximate at $\tau_{\mathrm{I}}=\tau$ or at $\tau_{\mathrm{D}}=\tau$. In the case $\frac{\theta}{\tau}>1$, both $\theta<$ 1 and $\tau<1)$ or $(\theta>1$ and $\tau>1)$, controlling worst case using PID parallel with derivative filter in $C^{\prime}(s)$ 
controller give the unstable respone with the big IAE value.

Step method of Mp-GM tuning is applied on 2DOFPID structure controller using approximation from 2DOF-IMC controller. The approximation step in $\mathrm{Mp}$ GM tuning allows the occurrence of deviation response that was resulted. This led to be produce a high overshoot response. But this overshoot will be reduced and the response can reach set point that is desired. To reduce a high overshoot that is produced from this method, can be added a correction factor that is multipied with gain controller value is produced from the approximation Mp-GM step method on 2DOF-PID.

\section{CONCLUSION}

A maximum peak-gain margin (Mp-GM) 2DOF IMC tuning method has used for two degree of freedom PID filter set point. The simulation results show that the maximum peak gain margin tuning method can give a good target set point tracking, disturbance rejection and robustness in system two degree of freedom PID structure controller with C'(s) controller using PID series with derivative filter. A high overshoot that was produced at this proposed method maybe caused from approximation step that is used to apply Mp-GM tuning metod at 2DOF-PID structure controller. To reduce a high overshoot response, maybe it can be added a correction factor that is multipied with gain controller value which is produced from this method. Future reserach is needed to formulate correction factor in order to reduce a high overshoot response that is produced.

\section{REFERENCES}

[1] H. M. Mazzini and D. F. G., Santos, "Two Degree of Freedom PID control for Integrating Process",XVIII Congreso Brasilleiro Automatica/12 a 16 Setembro 2010.

[2] M. Morari, and E. Zafiriou, Robust Process Control, Englewood Cliffs, NJ: Prentice -Hall, 1989.

[3] M. T. Tham "Part of a set of lecture notes on Introduction to Robust Control", Chemical and Process Engineering University of Newcastle Upon Time, 2002.
[4] D.E. Seborg, T.F. Edgar, and D.A. Mellicamp, "Process Dynamic and Control", $2^{\text {nd }}$ ed. USA: John Wiley \& Sons, Inc., 2004

[5] M. Araki, and H. Taguchi, "Two Degree of Freedom PID Controller", International Journal of Control, Automation and Systems, Vol. 1, No. 4, 2003.

[6] R. Vilanova, V. M. Alvaro, and O. Arrieta, "Analitycal Robust Tuning Approach for two degree of freedom", Engineering letter, 19:3,EL_19_3_08, 2011.

[7] I. Kaya, "Two-degree-of-freedom IMC structure and controller design for integrating processes based on gain and phase-margin specifications", IEE Proc.-Control Theory Appl., Vol. 151, No. 4, 2004.

[8] T.E. Marlin, "Process Control:Designing Processes and Control Systems for Dynamic Performance", Chemical Engineering Series. $2^{\text {nd }}$ ed. Boston: McGrawHill, 2000.

[9] H. Taguchi and M. Araki, "Two-Degree-of-Freedom PID controllers - Their functions and optimal tuning," in IFAC Digital Control: Past, Present and Future of PID Control, April 2000, Terrassa, Spain.

[10] D.H. Kim, "Tuning of 2-DOF PID controller by immune algorithm," In Congress on Evolutionary Computation (CEC'02), May 12-17, Honolulu, HI-USA, 2002, pp. 675-680.

[11] C. Brosilow and B. Joseph, "Techniques of model-based control", Prentice Hall International Series in the Physical and Chemical Engineering Science. New Jersey: Prentice Hall PTR 2001.

[12] K. Stryczek, M. Laiseca, C. Brosilow, and M.G. Leitman, "Tuning and Design of Single-Input, Single-Output Contro Systems for Parametric Uncertainty", AIChE Journal, Vol. 46, No.8, pp.1616-1631, 2000.

[13] Juwari, A. A. Badhrulhisham, C. S. Yee, and M. Rosbi "A New Tuning Method for Two-Degree-of-Freedom Internal Mode Control under Parametric Uncertainty", Process Systems Engineering And Process Safety. Chinese Journal of Chemical Engineering, Vol 21, No. 9, 2013.

[14] J. Zhang, J. Wang, and Z. Zhao, "A Novel Two Degree of Freedom PID Controller for Integrator and Dead Time Process", Proceedings of the $6^{\text {th }}$ World Congress on Intelligent Control and Automation, June 21 -23 2006, Dalian, China.

[15] R.Vilanova, "IMC based Robust PID design: Tuning guidelines and automatic tuning", J. of Proc. Cont. 18(1): 61-70 (2008).

[16] D.M. Chang, C.C. Yu, and I.L. Chien, "Coordinate control of blending systems", IEEE Trans. on Cont. Sys. Tech.. Vol. 6, No. 4, pp. 495-506, 1998

[17] J. Nancy and P.E. Sell, "Process Control Fundamentals for the Pulp and Paper Industry", TAPPI Process Control Textbook, Tappi Press, Atlanta, GA, 1995, pp. 428, 187. 


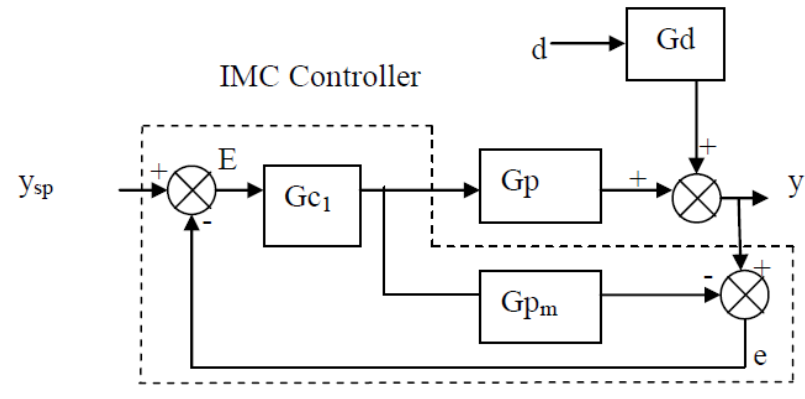

Figure 1. The structure of IMC controller
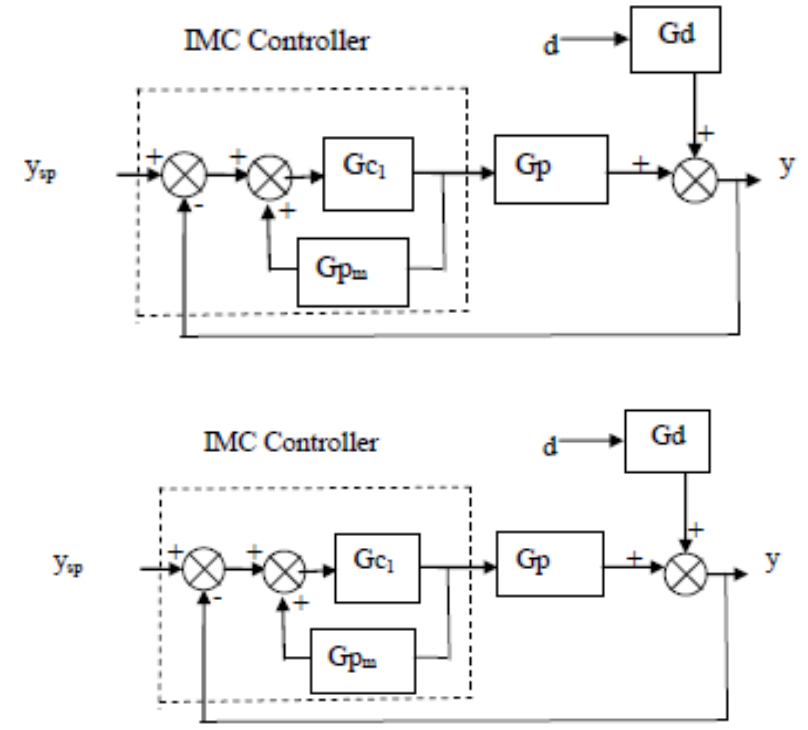

Figure 2. Simplified IMC controller to classical feedback control

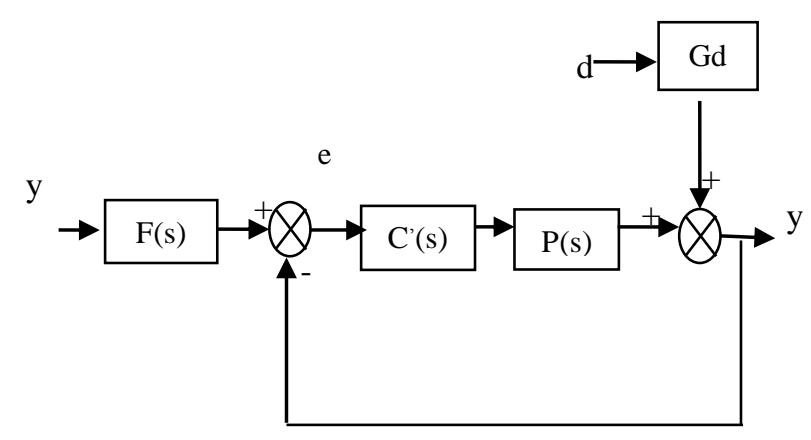

Figure 3. The structure of 2DOF-PID filter set point

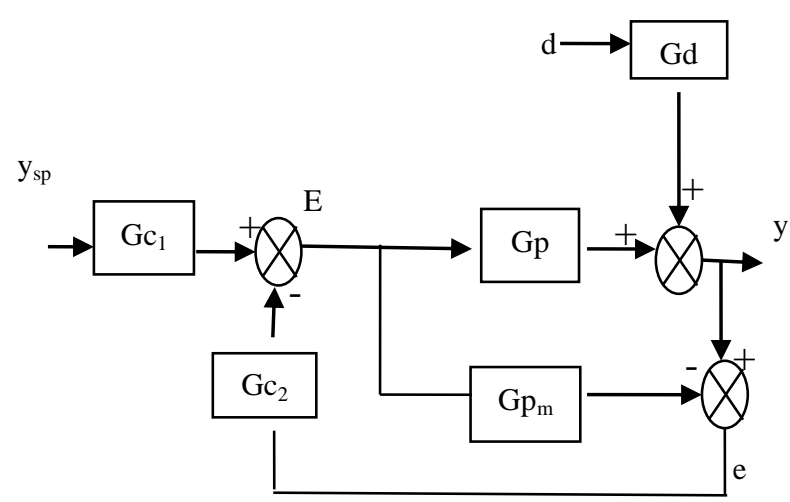

Figure 4. The structure of 2DOF-IMC standard

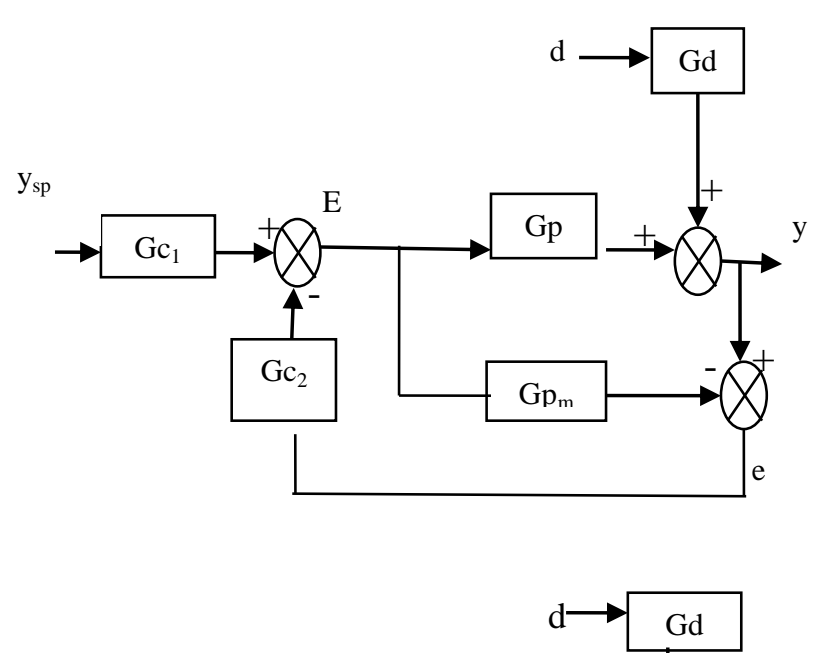

e

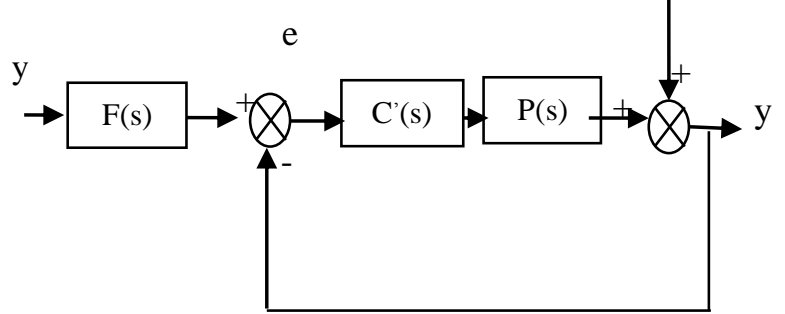

Figure 5. Simplified 2DOF-IMC controller to 2DOF-PID controller 


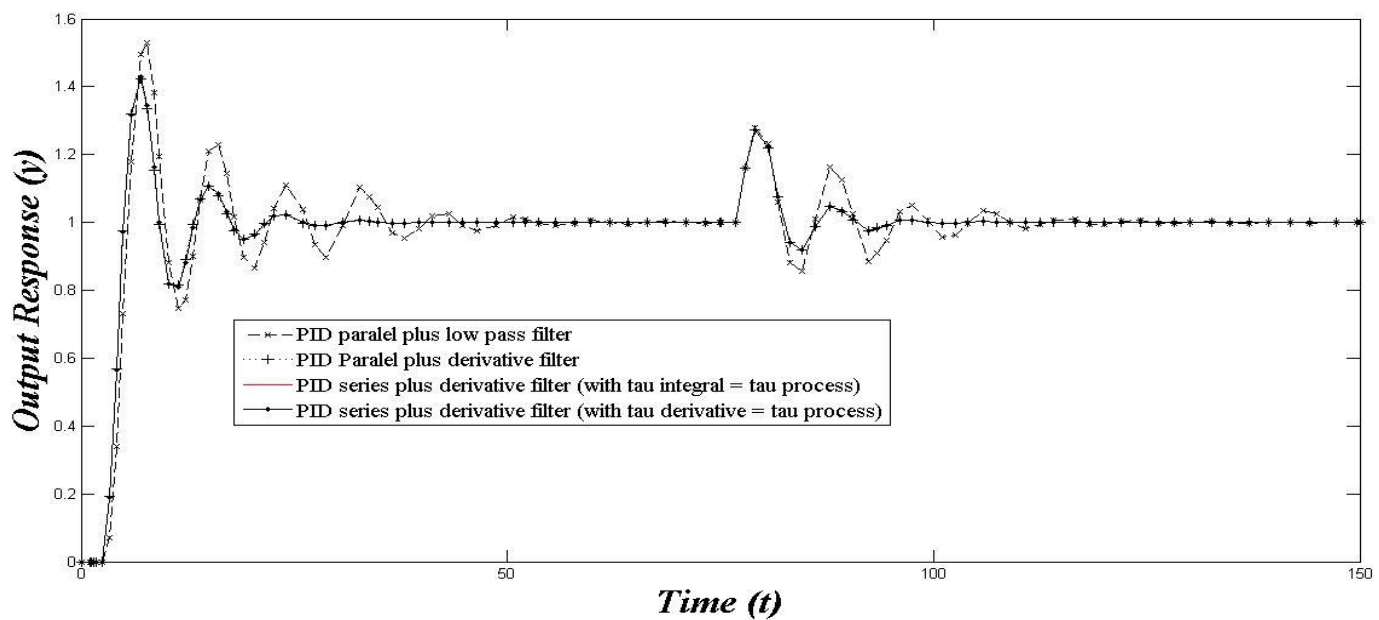

Figure 6. Output response for 2DOF PID structure control filter set point type with variation of PID controller design for C'(s) controller at the FOPDT case with $\frac{\theta}{\tau}<1$.

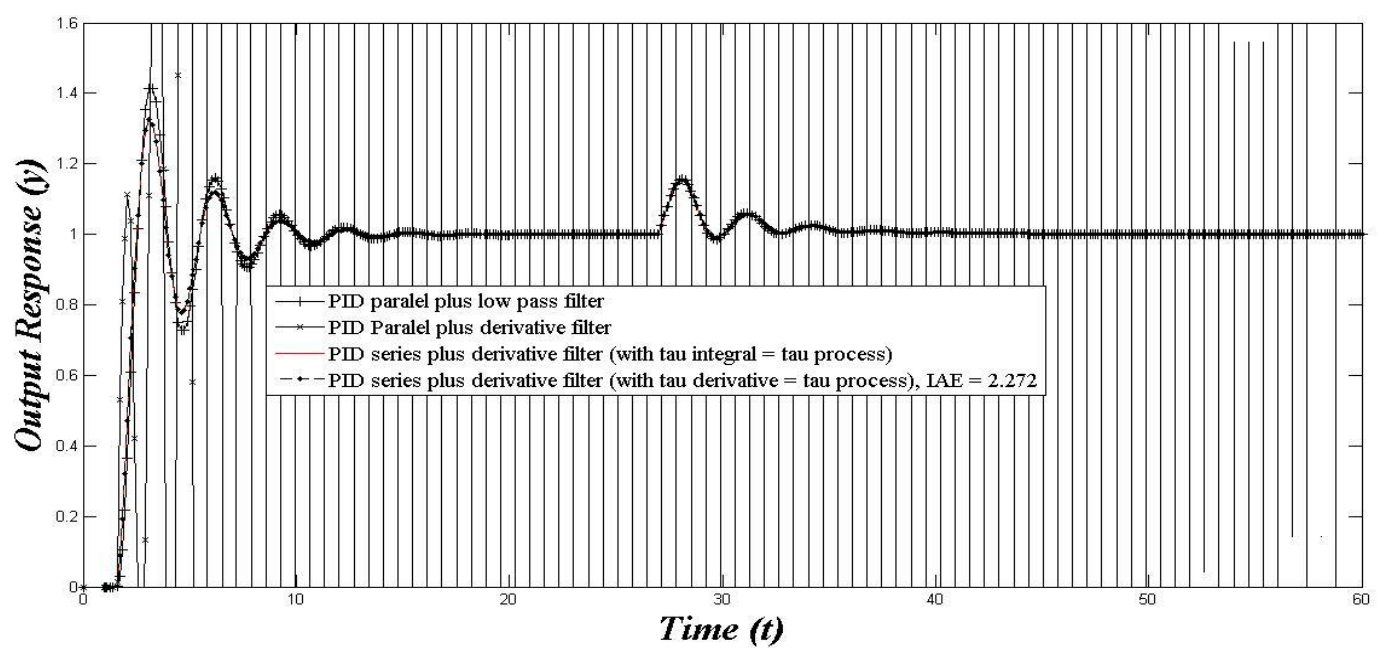

Figure 7. Output response for 2DOF PID structure control filter set point type with variation of PID controller design for C'(s) controller at the FOPDT case with $\frac{\theta}{\tau}>1$ (with $\theta<1$ and $\tau<1$ ).

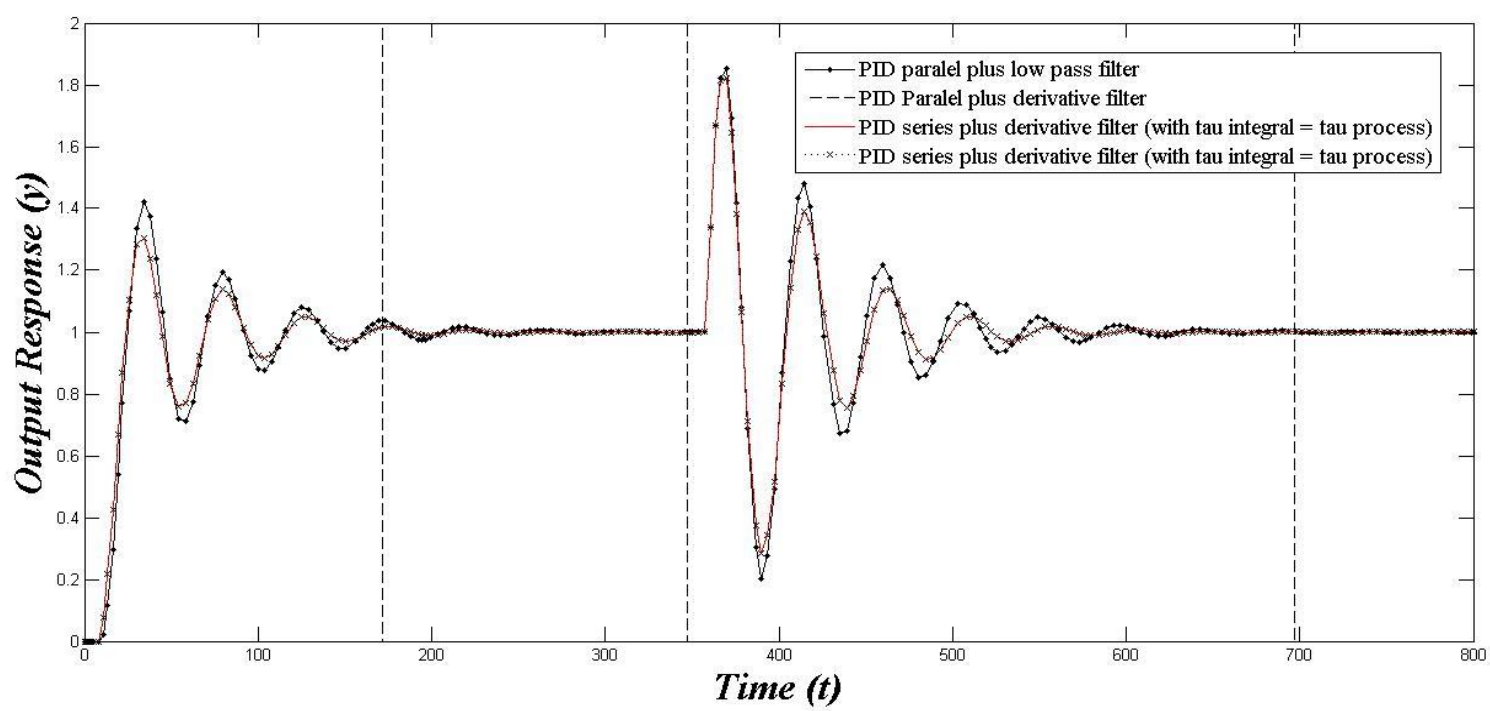

Figure 8. Output response for 2DOF PID structure control filter set point type with variation of PID controller design for C'(s) controller at the FOPDT case with $\frac{\theta}{\tau}>1$ (with $\theta>1$ and $\tau>1$ ) 
TABle 1.

Value of Controller Parametric for VARiety of Pid Controller on the Vilanova's Fopdt Case

\begin{tabular}{cccccc}
\hline PID controller Form & $\mathrm{k}_{\mathrm{c}}$ & $\tau_{\mathrm{I}}$ & $\tau_{\mathrm{D}}$ & $\tau_{\mathrm{f}} / \mathrm{A}$ & $\mathrm{IAE}$ \\
\hline Paralel plus low pass filter & 3.0674 & 6.0459 & 1.5114 & 3.8825 & 10.54 \\
Paralel plus derivative filter & 1.0976 & 2.1634 & 0.3413 & 9.8758 & 6.583 \\
Series plus derivative filter $\left(\tau_{\mathrm{I}}=\tau\right)$ & 1.5454 & 3.0459 & 3 & 1.2942 & 6.655 \\
Series plus derivative filter $\left(\tau_{\mathrm{D}}=\tau\right)$ & 1.5221 & 3 & 3.0459 & 1.2747 & 6.655 \\
\hline \hline
\end{tabular}

TABLe 2.

VAlue of Controller Parametric for Variety of Pid Controller on The Chang's Fopdt Case

\begin{tabular}{cccccc}
\hline PID Controller Form & $\mathrm{k}_{\mathrm{c}}$ & $\tau_{\mathrm{I}}$ & $\tau_{\mathrm{D}}$ & $\tau_{\mathrm{f}} / \mathrm{A}$ & IAE \\
\hline Paralel plus low pass filter & 0.1378 & 1.2216 & 0.1673 & 1.5989 & 2.594 \\
Paralel plus derivative filter & -0.0426 & -0.3774 & -2.1404 & -0.2564 & $2.055 \times 10^{27}$ \\
Series plus derivative filter $\left(\tau_{\mathrm{I}}=\tau\right)$ & 0.1153 & 1.0216 & 0.2 & 7.9949 & 2.272 \\
Series plus derivative filter $\left(\tau_{\mathrm{D}}=\tau\right)$ & 0.0226 & 0.2 & 1.0216 & 1.5652 & 2.272 \\
\hline
\end{tabular}

TABle 3.

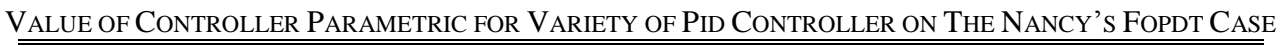

\begin{tabular}{cccccc}
\hline \hline PID Controller Form & $\mathrm{k}_{\mathrm{c}}$ & $\tau_{\mathrm{I}}$ & $\tau_{\mathrm{D}}$ & $\tau_{\mathrm{f}} / \mathrm{A}$ & IAE \\
\hline Parallel plus low pass filter & -124.4390 & 22.2997 & 3.1787 & 43.2133 & 80.97 \\
Parallel plus derivative filter & 116.7042 & -20.9136 & -46.6028 & 63.3886 & $2.176 \times 10^{32}$ \\
Series plus derivative filter $\left(\tau_{\mathrm{I}}=\tau\right)$ & -103.011 & 18.4597 & 3.84 & 11.2535 & 66.55 \\
Series plus derivative filter $\left(\tau_{\mathrm{D}}=\tau\right)$ & -21.4283 & 3.84 & 18.4597 & 2.3409 & 66.55 \\
\hline \hline
\end{tabular}

